\title{
Jean Gascou
}

\section{Unités administratives locales et fonctionnaires romains Les données des nouveaux papyrus du Moyen Euphrate et d'Arabie*}

Avant même notre première publication de cinq pétitions grecques l'an dernier (P. Euphr. 1-5) ${ }^{1}$, Fergus Millar a souligné l'intérêt historique unique des 21 nouveaux documents grecs et syriaques du Moyen Euphrate ${ }^{2}$. Outre 1-5, le monde savant dispose déjà des actes syriaques 19 et 20 édités par Javier Teixidor ${ }^{3}$. Les cinq actes de vente-achat (6-10) sont sous presse et paraîtront en principe au début de 1997 dans le Journal des Savants. Nous préparons pour 1997 ou 1998, toujours pour le Journal des Savants, l'édition de sept pièces publiables encore inédites (1117). Une pétition au bénéficier de police provenant de Bostra, datée de 260 (P. Bostra 1), dont je donne le texte ci-dessous, et un contrat de mariage contemporain de même provenance ( $P$. Bostra 2 ) devraient paraître prochainement. Je parlerai ici surtout du dossier syrien mais $\mathrm{P}$. Bostra 1 nous apportera quelques éléments de comparaison.

"Rome est la même partout" remarquions-nous dans notre introduction à P. Euphr. 1-5. La procédure de la pétition, telle que la décrivent ces cinq textes et P. Bostra 1, ne présente en effet rien qui s'écarte substantiellement de ce que nous

* Pour les abréviations des périodiques courants, on suit ici l'usage de l'Année Philologique. Pour les sigles papyrologiques, voir J. F. Oates, R. S. Bagnall, W. H. Willis, K. A. Worp, Checklist of Editions of Greek and Latin Papyri, Ostraca and Tablets (Atlanta ${ }^{41992) . ~ U n e ~}$ mise à jour permanente est diffusée sur Internet (http://odyssey.lib.duke.edu/papyrus/texts/ clist.html).

${ }^{1}$ Denis Feissel, Jean Gascou, Documents d'archives romains inédits du Moyen Euphrate (IIIe s. après J.-C.), I. Les pétitions (P. Euphr. 1 à 5), in: JS (1995) 65-119.

2 Fergus Millar, The Roman Near East 31 BC-AD 337 (Cambridge [Mass.], London 1993) 129-131, 155-156 et 478-481. Millar se fonde sur notre communication de CRAI (1989) 535561. Nos documents font l'objet de notices dans Hannab M. Cotton, Walter E. H. Cockle, Fergus Millar, The Papyrology of the Roman Near East: a Survey, in: JRS 85 (1995) 214-235. 3 Javier Teixidor, Deux documents syriaques du IIIe siècle après J.-C., provenant du Moyen Euphrate, in: CRAI (1990) 144-166 (19) et Un document syriaque de fermage de 242 après J.C., in: Semitica 41-42 (1991-1992 [1993]) 195-208 (20). Ces deux textes ont été réexaminés par Sebastian Brock, Some New Syriac Documents from the Third Century AD, in: Aram 3 (1991) 259-267. 
apprenaient déjà les papyrus égyptiens ou les inscriptions. Et même, surtout si nous considérons que le pouvoir romain, dans ce secteur, ne s'est consolidé qu'avec les Sévères, le milieu décrit par ce dossier paraît peut-être plus romanisé que l'Égypte: ainsi l'usage universel du denier, des dates consulaires, du calendrier romain à Nisibe et à Antioche, les nombreux gentilices et cognomina latins, les titres coloniaux portés par certaines villes (Antioche, Carrhes, Nisibe), les institutions municipales de Marcopolis et de Carrhes, les références explicites ou implicites au droit romain 4 . Pourtant, quand on entre dans le détail des textes dits du Moyen Euphrate, on ne peut manquer de nuancer cette vue.

Au préalable, il convient d'évoquer le problème, que nous n'avons pas encore véritablement résolu, de la provenance du dossier: cette demi-incertitude en obère en effet la mise en perspective.

\section{Provenance du dossier}

Il s'agit d'une trouvaille irrégulière remontant au plus tard à 1987. D'après l'acheteur et propriétaire actuel, le marchand aurait évoqué lors de la transaction les environs de Doura, ce qui est possible, mais pas garanti. Le lot est homogène. Outre les textes grecs et syriaques sur papyrus et sur peau, il comprenait de menus objets de la vie quotidienne (bois tourné, poterie, verrerie, vanneries etc.). Deux textes trop fragmentaires ou détériorés sont impubliables et nous n'avons aucune idée de leur nature. Les pièces datées ou datables se distribuent sur une période très courte, les années 232 à 252 et sont plus nombreuses dans la deuxième décennie de la tranche chronologique envisagée que dans la première. Elles sont surtout juridiques, en ce sens qu'elles se rapportent à des litiges et à des transactions. Deux documents privés seulement, des lettres, encore ces deux derniers textes ont-ils tous les traits des lettres d'affaires: il y est surtout question d'argent et de gestion économique. Ces caractères, l' association avec des objets domestiques, la concentration chronologique, la prédominance des papiers juridiques, apparentent typologiquement le dossier aux trouvailles textuelles de Murabba'at, de Nahal Hever (constituées principalement par les archives dites de Babatha ou P. Yadin), ou de Transylvanie. Nous avons affaire à ce qu'Alain Martin appelle des cachettes documentaires ${ }^{5}$, à ces dépôts, analogues aux trésors monétaires, qui se constituent à des époques troublées, où les particuliers, dans l'attente d'un retour à la normale qu'ils espèrent rapide, mettent à l'abri non seulement leurs personnes et leurs écono-

4 Ainsi dans l'acte inachevé 15, rédigé au village de Beth Phouraia en 235, Aurelia Barabous,

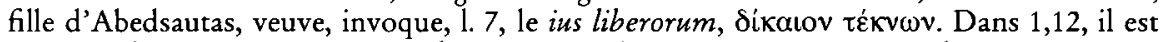
question des constitutions impériales ( $\left.\theta \varepsilon \tilde{\alpha} \alpha \iota \delta \iota \tau \alpha^{\alpha} \xi \varepsilon \iota \zeta\right)$ et nous avons noté dans cette pièce et dans 2, des allusions à l'Édit du Préteur.

5 Alain Martin, Archives privées et cachettes documentaires, in: Proceedings of the 20th International Congress of Papyrologists (Copenhagen 1994) 575, comparant notre dossier avec les P. Yadin et les tablettes de Transylvanie. 
mies, mais aussi leurs papiers les plus importants comme leurs titres de propriété (notamment chez nous ceux de leurs esclaves ou de leurs chevaux) ou les actes relatifs à leurs affaires en cours (prêts; deposita; procès) ${ }^{6}$. Dans le cas de Murabba'at, de Nahal Hever et des tablettes de Transylvanie, les circonstances sont claires: la révolte de Bar Kochba sous Hadrien, les guerres daciques sous Marc Aurèle. En ce qui regarde le dossier du Moyen Euphrate, la cachette est certainement liée à l'offensive sassanide de 256 qui, comme pour Doura, donne le terminus ante quem. Quant aux lieux, après avoir hésité quelque peu à cause de la variété d'origine des papiers et notamment de leur importante composante mésopotamienne, nous sommes sûrs du moins que, comme Doura, la région d'origine relevait de la province romaine de Coelè Syrie, puisque les habitants du secteur étaient sous la juridiction d'un consulaire, íđatıkó leurs procès (1). D'autre part, d'après 5 et 14 , la région était sous la protection de détachements de la legio XVI Flavia Firma, et de la cohors XX Palmyrenorum ${ }^{7}$, qui sont des unités syriennes déjà bien connues entre autres par les papyrus de Doura. Les militaires sont en fait omniprésents dans le dossier. Ils constituent l'épine dorsale de la société. J'évoquerai ici leur rôle administratif, mais leur rôle social et économique mériterait une étude.

Quelque vingt toponymes figurent au dossier. Certains apparaissent ponctuellement dans des contextes d'affaires ou de procès, ainsi en Coelè Syrie, Antioche, Zeugma, Beroia, Dusaris et peut-être Reșāfāa en Syrie Phénicie. Il en va de même pour les grands centres d'Osrhoène et de Mésopotamie qui sont mentionnés à loccasion d'achats d'esclaves (Marcopolis; Nisibe) ou d'une jument (Carrhes), d'une prise d'hypothèque, d'une créance (Marcopolis). Sans contribuer à la localisation, ces données attestent surtout la grande mobilité des propriétaires de nos papiers, ou l'étendue de leurs intérêts. Nous savons d'ailleurs que certains de ces personnages étaient des caravaniers, des pasteurs, des commerçants souvent sur les routes: ainsi dans la lettre 16, un certain Ourôdès fils de Nisraios évoque ses bergers, son chamelier, le taux de location des chameaux entre Béroia et Zeugma, 36 deniers par kor. Dans une autre lettre, où il est question de grosses sommes d'argent (17), Roumas avertit un homonyme de ses étapes, Dusaris et Ressa(fa?). D'autres lieux, les plus cruciaux pour notre problème, Beth Phouraia (le plus fréquemment mentionné), Magdala, Birtha Okbanôn et Appadana, restent à fixer sur

${ }^{6}$ Le bon état de conservation de la plupart des documents suggère que des précautions furent prises pour les préserver de la détérioration naturelle, notamment de l'humidité, fatale aux écrits.

7 Voir en dernier lieu David L. Kennedy, The Cohors XX Palmyrenorum, at Dura Europos, in: The Roman and Byzantine Army in the East (Krakow 1994) 89-98.

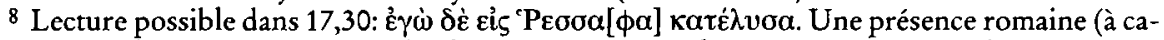
ractère militaire) est attestée archéologiquement à Reșāfā pour notre époque (deuxième tiers du IIIe s.); voir Michaela Konrad, Flavische und spätantike Bebauung unter der Basilika B von Resafa-Sergiupolis, in: Damaszener Mitteilungen 6 (1992) 313-402, sp. 347-348. Des monnaies sont venues s'ajouter aux indices recueillis par Konrad (communiqué par Pierre-

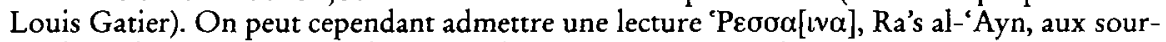
ces du Hābūr, ce qui s'accorderait avec les vues exprimées ci-dessous. 
la carte. Appadana, dont dépendent administrativement Beth Phouraia, Birtha et Magdala, paraît le dénominateur commun du dossier. C'est de la région d'Appadana que provenaient nos fugitifs, et c'est là peut-être qu'ils se réfugièrent vers 256 et constituèrent la cachette. Appadana est mentionné dans les papyrus de Doura et chez nous comme siège d'un procurateur, d'une vexillatio de la cohors XX Palmyrenorum $^{9}$, et aussi comme centre administratif d'une région ou d'une voie appelée la praetentura. La pétition 5 suggère que c'était le chef-lieu d'un district de police appelé la Sphoracène ou encore que la Sphoracène faisait partie des districts du territoire d'Appadana. Il y avait en Orient plusieurs Appadana, l'un d'entre eux ayant survécu dans le nom de la ville iranienne d'Ibadan ou Abadan. Le nôtre n'est pas encore identifié et nous n'avons pas pris de position personnelle sur ce point dans la présentation que nous avons faite du dossier en 1989 devant l'Académie des Inscriptions et Belles-Lettres. Il est toutefois à peu près assuré par P. Dura 60 (CEL 178) qu'Appadana se trouvait en amont de Doura. Certains spécialistes le placent hypothétiquement sur la rive droite de l'Euphrate ${ }^{10}$. D'autres le rapprochent du site antique de Tall Fudayn sur la rive droite du Bas Hābūr, l'affluent oriental de l'Euphrate, ce qui serait étymologiquement plausible sans vraiment contrevenir aux données de P. Dura 6011. Fudayn n'a pas encore été fouillé et laisse surtout voir des vestiges islamiques, mais de la céramique et une monnaie romaines ont été recueillies en surface ${ }^{12}$. Il est à noter aussi que 8 et 9 font état d'un district d'Abourène associé à trois agglomérations, Theganaba, Bèathagaé et $\mathrm{Ba}$ nathsamsa. Le nom de l'A bourène comporte une allusion claire au Hābūr et, grâce à des sources byzantines et médiévales, nous avons pu situer Banathsamsa dans le diocèse de Dara: deux raisons laissant à penser que cette Abourène enveloppait notamment le moyen cours du Hābūr, autour de l'actuel Hasaka, ce qui nous re-

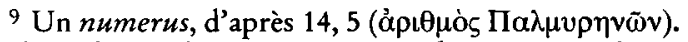

10 En dernier lieu Marie-Louise Chaumont, Un document méconnu concernant l'envoi d'un ambassadeur parthe vers Septime Sévère (P. Dura 60 B.), in: Historia 36 (1987) 422-447, suivant Mikhail Rostovtzeff, Les archives militaires de Doura, in: CRAI (1933) 321.

11 Fudayn (malgré des variantes vocaliques comme Fidēn) est morphologiquement un diminutif arabe (fu'ayl), comme on en trouve d'autres exemples en toponymie proche-orientale actuelle (ainsi Dumayr, Bușayra, Kuwayt; voir Thomas Bauzou, Journées de travail „inscriptions grecques et latines de la Syrie“ (avec la Jordanie), in: Syria 70 [1993] 41). Cette forme est utilisée notamment pour transposer des toponymes antiques (Suwayda pour Soada; 'Anayba pour Nab, via une vieille forme arabe Onevatha qui était déjà un fu'ayl selon Bauzou, art. cit.). Fudayn peut fort bien correspondre à un ancien Appadana. Pour les savants de cette opinion, voir Wolfgang Röllig, Hartmut Kübne, The Lower Habur, a Preliminary Report on a Survey conducted by the Tübinger Atlas des Vorderen Orients, in: Les Annales Archéologiques Arabes Syriennes 27/28 (1977-1978) 120 n. 31 (aimablement communiqué par Michael Fuller).

12 Voir Antoine Poidebard, La trace de Rome dans le désert de Syrie, (BAH 1934) 134, décrivant Fudayn comme une „ville assyrienne“ avec des restes de murs arabes et byzantins, ayant d'avion l'aspect d'une ville fortifiée, avec plusieurs plates formes étagées autour d'un tall central, citadelle de la place, donnant le type classique des villes fortes du Bas Hābūr; Röllig, Kübne, in: Les Annales Archéologiques Arabes Syriennes 27/28 (1977-1978) 120-121; Hartmut Kübne, Zur historischen Geographie am Unteren Huābūr, in: AOF 25 (1974/1977) 249255, avec plan, 251 ; photographie du site par le même auteur in: AOF 26 (1978/1979) 186. 
porte encore vers la Mésopotamie géographique. D'un autre côté, un des villages du ressort d'Appadana, Beth Phouraia, est décrit dans 20 comme étant „sur l'Euphrate" (DBPRT). Si nous rassemblons toutes ces données, il semble que notre dossier émane d'une région syrienne à forte présence militaire, située au confluent de l'Euphrate et du Hābūr, très tournée vers l'Osrhoène et la Mésopotamie et dont le centre administratif s'appelait Appadana. Et c'est autour de l'étude de cet ensemble que j’organiserai la suite de cet exposé.

\section{Les ressorts régionaux}

Si l'hypothèse précédente sur Appadana se confirmait, il faudrait admettre que la province de Coelè Syrie n'était pas en ce point limitée par l'Euphrate, mais qu'elle débordait sur la rive mésopotamienne du fleuve le long du Hābūr. Nous avions déjà des indices en ce sens par des papyrus de Doura ${ }^{13}$. L'identification proposée récemment entre le castellum arab () des P. Dura 100 et 101 et les toponymes actuels de 'Arabān / 'Arbān / Tall 'Ağağa sur le Bas Hābūr qui semblent correspondre au site antique d'Oraba ( ou Oroba, Horaba) attesté par la Notitia Dignitatum, Or. 35, serait de nature à confirmer cette vue ${ }^{14}$. À l'époque byzantine ce territoire sera incorporé avec peut-être l'Abourène ${ }^{15}$ et Oraba à la nouvelle province d'Osrhoène. Issu en partie d'un démembrement de la province sévérienne de Mésopotamie, ce ressort était limité à l'est et à partir de Circesium, au confluent de l'Euphrate, par le Hābūr qui le séparait de l'empire perse. La limite entre l'Osrhoène et la Mésopotamie byzantines est mal connue, en raison, en particulier de corruptions dans les passages pertinents de la Notitia Dignitatum ${ }^{16}$. On serait cependant tenté de l'établir sur le haut cours du Hābūr, jusqu'à Ra's al-'Ayn (Re-

13 P. Dura 25, 5, 21, d'époque romaine, mentionne le village de Nagabata sans doute le futur Circesium (ChLA VI 315, p. 27, n. 10), dont Isidore de Charax indique que le Hābūr la longe (voir P. Dura, p. 111). Dans P. Dura 26, de 227, une terre achetée par un vétéran est limitée à l'est par le Hābūr. Voir P. Dura, p. 24; Cotton, Cockle, Millar, Papyrology 215.

14 Les $P$. Dura 100 et 101 ne permettent pas d'aller plus loin dans la résolution de la forme que castellum arab( ). Pour la localisation du toponyme, voir Antonio Invernizzi, KifrinBНХХОҮФРЕIN, in: Mesopotamia 21 (1986) 64 et Fabrizio A. Pennacchietti, Il posto dei Cipri, in: Mesopotamia 21 (1986) $88 \mathrm{n}$. 15. Le dernier auteur propose une lecture Arabionis au lieu de l' Arabum de l'éd. pr. des P. Dura, p. 40. Pennacchietti s'appuie sur la mention d'un

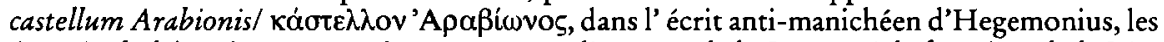
Acta Archelai, qui se trouverait sur un cours d'eau appelé le Stranga, à la frontière de la Mésopotamie et de l'empire perse (voir du même Pennacchietti, Gli Acta Archelai e il viaggio di Mani nel Bēt 'Arbāyēê, in: RSLR 24 [1988] 503-514). Bien que les données narratives des Acta aient généralement peu de crédit, en particulier dans l'ordre toponomastique (il n'est pas si simple d'identifier, avec Pennacchietti, le Stranga au Häbūr), la coïncidence entre les Acta et les P. Dura est troublante.

15 A notre Banathsamsa et à notre Theganaba, pourraient correspondre Banasam et Ganaba d'Osrhoène ( Not. Dig., Or. 35).

16 Or. 35 (Osrhoène) et 36 (Mésopotamie). 
saina / Théodosiopolis $)^{17}$. Une vignette de la Notitia Dignitatum représente en Osrhoène (Or. 35) un centre de garnison appelé Apatna qui pourrait bien coïncider avec notre Appadana ${ }^{18}$. Le Magdala des $P$. Dura et peut-être de 5 (le Magdalatha de Procope, Aed. II, 6, 14?) correspondrait au Tall Mağdal du Haut Hābūr, ce qui situerait la Sphoracène autrement que nous ne l'avons fait dans notre édition de $5^{19}$. Le village de Dusaris (14 et 17) ne serait pas en ce cas le Dausara de Ptolémée sur l'Euphrate, en amont de Nicephorium, mais le Dausara mésopotamien de Procope (Aed. II, 6, 14). Encore que parsemée de sites antiques ${ }^{20}$, la vallée du Huābūr, n'était pas une région de grandes villes. Outre ses activités agricoles, elle ménageait surtout un passage commode entre la vallée de l'Euphrate et la Mésopotamie du Nord (Nisibe) ${ }^{21}$. Cette voie pouvait être utilisée par les Perses pour prendre l'Osrhoène à revers: c'est un projet de ce genre, visant Batnai, que décrit Ammien Marcellin, Hist. XIV,3. Les Saracènes ajoutaient leur menace. Rome puis Byzance paraissent donc avoir solidement gardé les rives de notre rivière22.

$\mathrm{Si}$ le dossier provient bien de ces contrées transeuphratènes, on s'expliquerait mieux ainsi le statut administratif assez singulier du ressort d'Appadana dans nos documents. Tout d'abord, d'après les pétitions 1 et 2 , Appadana était vers 245 le siège d'un procurateur de rang egregius appelé Claudius Ariston. Nous n'avons pas réussi, jusqu’à présent, à nous faire une doctrine sur ses attributions. Les deux pétitions que nous venons de citer montrent que ce personnage exerçait, par délégation du consulaire de Syrie, une activité juridictionnelle civile. Mais était-ce là sa fonction essentielle? Un de nos documents (1) le dit aussi responsable de la

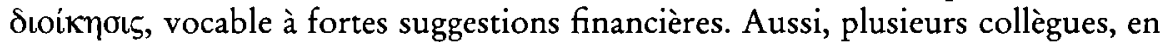
particulier Rudolf Haensch, sont-ils d'avis de reconnaître en Ariston un procurateur financier provincial ${ }^{23}$. Haensch fait en particulier remarquer que les pétitionnaires de 1 sont originaires du village "impérial“ de Beth Phouraia, le lieu-dit le

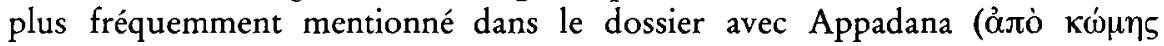

17 Voir Louis Dillemann, Haute Mésopotamie orientale et pays adjacents (Paris 1962) 105110.

18 Rien dans le texte de Not. Dig., Or. 35 ( $d u x$ Osrboenae), ce qui oblige à supposer une lacune, à moins que les données correspondantes ne soient passées à la notice concernant la Mésopotamie, qui paraît perturbée (Not. Dig., Or. 36).

19 JS (1995) 114-116 (nous inclinions pour un secteur du Moyen Euphrate).

20 Pour des descriptions anciennes de la vallée du Hābūr, voir Michel Tardieu, Les paysages reliques, routes et haltes syriennes d'Isidore à Simplicius (Louvain-Paris 1990) 104-105.

21 Benjamin Isaac, The Limits of Empire, the Roman Army in the East (Oxford 21992) 255256. Nos textes 8 et 9 offrent une bonne illustration de cette vocation de l'Abourène par où transitaient des esclaves de Nisibe destinés à être revendus à Beth Phouraia.

22 Les fouilles récentes, particulièrement celles de Tall Tunaynīr (antique Thannouris ?) confirment la présence romaine sur le Hābūr. Le Prof. Michael Fuller m'informe ainsi aimablement, par lettre du 15 août 1996, de la découverte sur ce site de monnaies d'Antonin le Pieux et de Philippe l'Arabe, de céramique du IIe siècle. Une statue de marbre acéphale d'un empereur romain (Septime Sévère?) a été mise au jour en mars 1996 près de Ra's al-'Ayn. Ces trouvailles ne tranchent malheureusement pas la question des ressorts.

${ }^{23}$ Rudolf Haensch, Capita provinciarum (Mainz 1995) 285, n. 139 (non vidi mais les épreuves du lieu pertinent de cet ouvrage m'ont été aimablement communiquées par l'auteur). 


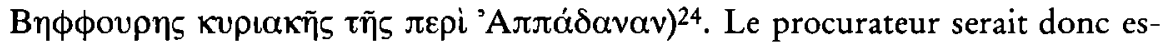
sentiellement un agent chargé de gérer les revenus tirés des domaines impériaux, des praedia fiscalia déjà attestés en effet dans ce secteur du Moyen Euphrate ${ }^{25}$. Pourtant, le pétitionnaire de 2 ne dit pas que son lieu d'origine, Birtha Okbanôn, appartienne aux domaines impériaux et lorsqu'il se réfère à Claudius Ariston, il n'emploie que l'expression „le procurateur d'Appadana“. C'est pourquoi, nous n'excluons toujours pas que ce procurateur n'ait été chargé spécialement de l'administration de cette partie de la Syrie. La dioikèsis, d'après des exemples empruntés à l'Asie Mineure, aurait ici le sens de circonscription judiciaire, conventus ${ }^{26}$.

Au problème d'Appadana et de son procurateur est lié celui de la praetentura.

Nous savions déjà par $\mathrm{P}$. Dura $64 \mathrm{~A}, \mathrm{v}^{\circ}, 3$ et $\mathrm{B}, \mathrm{v}^{\circ}, 3^{27}$ que dès 221 , Appadana dépendait d'un dispositif appelé praetentura, soumis à un procurateur directement soumis au consulaire de Syrie appelé praepositus praetenturae ${ }^{28}$. Cet agent se retrouve entre 252-256 dans nos n's 3-4, pétitions adressées au praefectus et praepositus de la praetentura, un certain Julius Proculus. Cette notion de praetentura ainsi que les vocables apparentés comme praetensio ou protensio est obscure. L'étymologie et les contextes disponibles évoquent un dispositif militaire protecteur avancé. Dans P. Dura 64, le praepositus praetenturae est amené, sur ordre du gouverneur provincial, à s'occuper du ravitaillement en fourrage de la vexillation d'Appadana (les Palmyréniens). Ammien Marcellin à propos de la Mésopotamie, met praetentura sur le même plan que statio agraria, ce qui suggèrerait une acception ponctuelle, sans toutefois l'imposer ${ }^{29}$. D'autre part, comme Ammien indique qu'on trouvait des praetenturae dans tous les districts de la Mésopotamie (tractus omnes), il ne semble pas qu'il faille donner nécessairement à ce mot le sens de $l i$ mes, ou de ligne de fortification frontalière comme l'expliquent les éditeurs de P. Dura 64 („some part of the limes“). D'après CIL VIII 22062-64, où il est question de la pose des milliaires de la nova praetentura de Maurétanie Césarienne sous Septime Sévère et Caracalla, une praetentura pourrait être une route militaire, ou une ligne de communication fortifiée, sens qui pourrait convenir aussi à la praetentura Italiae et Alpium, qu'on voit soumise à un légat impérial vers 168 pendant une expédition contre les Germains ${ }^{30}$. De même pour la praetensio d'une ins-

${ }^{24}$ Ce statut de Beth Phouraia est attesté par deux autres de nos pièces, 9,7 et 11,4-5; il manque dans 3-4, 8, 12 et 15 .

25 P. Dura $64 \mathrm{~A}, \mathrm{i}, 11$ et p. 230.

${ }^{26}$ Haensch, ibid., admet que ce sens est possible. Pourquoi refuser a silentio l'existence d'un système de conventus en Syrie, surtout si on garde à l'esprit que la pétition 1 évoque une

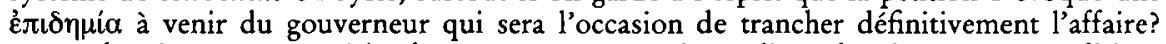
Haensch estime que cette visite du gouverneur pourrait être liée à des circonstances politicomilitaires exceptionnelles (la reconquête de la Mésopotamie) que le texte n'évoque nullement. 27 Fink 91, ChLA VI 319.

28 Vu la manière dont ce procurateur s'adresse aux autorités militaires de Doura (demande polie justifiée par un ordre du consulaire et non pas ordre), Doura ne paraît pas avoir fait partie à coup sûr du dispositif.

29 Hist. 14,3,2.

30 I.Lat. Alg. II, 2,4681 (autant que „eine vorgeschobene Kriegszone unter Militärverwal- 
cription tétrarchique d'Azraq en Arabie. Le texte est mutilé au début ce qui rend d'interprétation difficile la junctura praetensione coligata des 1. 4-531. Mais il est peut-être significatif que la suite du document fasse état d'un millage de Bostra à Dumata.

D'après 3-4, la praetentura est aussi un ressort de police et non pas simplement militaire: le praepositus peut déférer à la justice du gouverneur de Syrie des parties en conflit. Il a donc des atttributions civiles, si bien qu'à titre d'hypothèse, on se demandera si le procurateur d'Appadana évoqué par nos $\mathrm{n}^{\circ} \mathrm{s} 1$ et 2 ne se confond pas en fait avec le praepositus praetenturae, dont la qualité de procurateur est attestée en effet par P. Dura 64.

\section{Les ressorts administratifs locaux: continuités et transformations}

De ce point de vue, on peut diviser le dossier en deux groupes: les pièces „épichoriques" centrées sur Appadana et les textes mésopotamiens ou comportant des allusions à des centres mésopotamiens et que je ne cite que pour mettre en valeur les contrastes institutionnels et culturels entre les régions décrites. Il s'agit donc directement ou indirectement de Carrhes, Marcopolis et Nisibe (19, 20, 6-7, 8, 9 et 10). Dans le groupe documentaire concerné, on saisit, conformément à l'image que se formaient les Anciens de la Mésopotamie, une puissante vie urbaine et municipale, comme nous le montrait déjà pour Édesse P. Dura 28. Nous assistons ainsi, entre 240 et 249, avec 19,20, et 6-7, à la disparition définitive du royaume client des Abgarides et, corrélativement à la naissance ou à la renaissance des institutions civiques de Marcopolis, avec ses sacerdoces ${ }^{32}$, ses duoviri municipaux (les „archontes" d'après les souscriptions syriaques de 6-7), ses archives (ả $\rho \chi \varepsilon \tilde{\alpha} \alpha$ d'après 6-7). Comme en Arabie après 106, le parler araméen local encore utilisé dans 20 en 242, fait place au grec dès 249 (6-7). Carrhes et Nisibe apparaissent comme des colonies et métropoles, la colonie de „Septimia Nisibe“ ayant même adopté le calendrier romain (8). Carrhes a ses tribus municipales et utilise officiellement le grec (10). Inversement, dans le premier groupe, le groupe syrien, ce qui frappe, c'est le faible développement des institutions municipales. Les seuls ressorts locaux définis sont appelés des „villages“, $\kappa \tilde{\omega} \mu \alpha$ (ainsi Beth Phouraia, Mag-

tung“; Jaroslav Šašel, Über Umfang und Dauer der Militärzone Praetentura Italiae et Alpium zur Zeit Mark Aurels, in: MH 31 [1974] 226; voir aussi Neil Cbristie, The Alps as a Frontier (A.D. 168-774), in: JRA 4 [1991] 412-413).

31 David L. Kennedy, Henry I. MacAdam, Latin Inscriptions from the Azraq Oasis, Jordan, in: ZPE 60 (1985) 97-107, sp. 100-104 ( AE 1987, 963); voir Michael P. Speidel, The Roman Road to Dumata (Jawf in Saudi Arabia) and the Frontier Strategy of praetensione colligare, in: Historia 36 (1987) 213-221, et l' importante discussion de Constantin Zuckerman, Aur. Valerianus (393/305) et Fl. Severinus (333), in: Antiquité Tardive 2 (1994) 83-88, sp. 86-87, d'où semble ressortir l'idée de route militaire. Aussi Isaac, Limits of Empire 169.

32 Voir la réédition partielle de 20, par Brock, in: Aram 3 (1991) 268-271 et 268 n. 2. 
dala, Banathsamsa, Béathagaè, Dusaris) ${ }^{33}$. Lorsque les habitants de tel de ces villages pétitionnent auprès du gouverneur de Syrie ou auprès d'autres instances habilitées à recevoir des libelles, ils se dispensent parfois (comme en 2) d'indiquer leur chef-lieu ${ }^{34}$ ou encore ils donnent simplement le canton de rattachement, ainsi

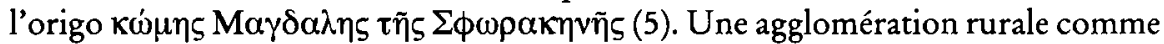

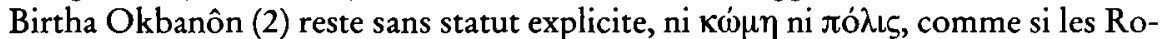
mains lui refusaient la qualité de collectivité de droit public. Même absence de définition pour les districts, ainsi la Sphoracène, qui, d'après 5 , paraît officieusement faire partie du territoire administré par Appadana où elle formait une circonscrip-

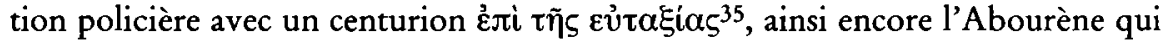
dépendait de Theganaba au titre de sa $\pi \varepsilon p i ́ x \omega \cos ^{36}$. Il serait pourtant tentant de reconnaître dans cette Sphoracène, dans cette Abourène, l'une ou l'autre de ces antiques subdivisions de la Mésopotamie préromaine que L. Dillemann appelle des "cantons" et qu'il définit comme les "plus petites divisions territoriales connues, groupées autour d'une ville dont elles prennent généralement le nom". Les exemples cités par Dillemann comportent de nombreuses formations en - $\eta v \eta$ (Osrhoène, Anzitène, Zabdicène etc.) analogues aux nôtres ${ }^{37}$. De tels cantons s'observent aussi en Arabie d'après les P.Yadin (ainsi la Zoarène, qui était un canton de la cité de Pétra situé au sud de la Mer Morte) et dans P. Bostra 1: ce texte fait en effet état d'un ressort appelé l'Aianitide, qui était une ancienne N $\alpha \beta \alpha \tau \alpha i \omega v$ $\chi \omega ́ p \alpha$ selon l'historien ou ethnographe hellénistique des Arabes Ouranios. Incorporée au territorium de Bostra par les Romains, cette Aianitide y a gardé une certaine consistance, notamment pour la police puisqu'elle avait en 260 un beneficiarius particulier: forte ressemblance avec notre Sphoracène. Il semblerait donc que les Romains aient pris en charge avec les "cantons" une géographie administrative bien antérieure à leur installation.

Même imprécision pour les chefs-lieux, c'est-à-dire Theganaba, et, ce qui est plus frappant, Appadana lui-même, dont nous venons de mesurer toute l'importance administrative et militaire. Jusque aux années 252 , ce centre est resté sans statut communautaire reconnu. Son nom, d'origine iranienne, signifie résidence royale ( $\beta \alpha \sigma i \lambda \varepsilon\llcorner\alpha)$, ou siège d'un satrape. Les Romains ont pu trouver commode d'installer leurs fonctionnaires et leurs soldats à un endroit traditionnellement voué à servir de siège à une autorité, continuité qu'ils n'ont pas tout de suite cherché à sanctionner par des définitions juridiques. C'est après 252 seulement et avant l'invasion sassanide de 256, au moment où Doura devient colonie, qu'Appadana, changeant son nom en celui de Néapolis, devient une cité romaine dotée d'un sénat (3-4). Avec une dizaine d'années de retard, Appadana partage ainsi le sort de

33 Aucune institution villageoise n'est cependant mentionnée.

${ }^{34}$ De même dans l'acte syriaque 20, écrit en Osrhoène, l'origo de Wôrôd, un Syrien de Beth Phouraia, est simplement „du village de Bet Purai sur l'Euphrate “, imprécision record.

${ }^{35} \mathrm{Ce}$ titre, inconnu en Égypte, réapparaît dans P. Bostra 1.

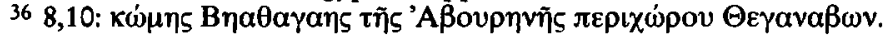

37 Dillemann, Haute-Mésopotamie 102-103, admettant qu'on sait peu de choses sur l'organisation interne de ces circonscriptions. 
l'ancienne résidence royale abgaride de Hayklā-Karkā de Sīdā transformée entre 240 et 242 en municipe de Marcopolis (19, 20, 6-7). Il semble que jusque là, l'administration romaine ait administré directement ces contrées, par le biais du fisc, dans le cas des villages impériaux comme Beth Phouraia, des procurateurs d'Appadana ( 1 et 2 ), du praepositus praetenturae qui se confond peut-être en fait comme nous l'avons vu, avec le personnage précédent (3-4), ou des centurions

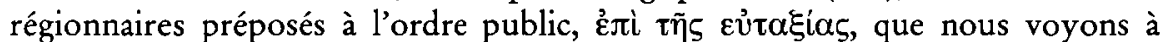
l'oeuvre dans 2 et 5. C'est sans doute l'officier de police qui apparaît en 15,2-3: un habitant de Beth Phouraia apprend à son fils qu'il s'est rendu accompagné auprès $\mathrm{du}$ „porte épée“ et que ce dernier les a reçus et regardés d'un bon oeil: عiới $\lambda \theta 0 \mu \varepsilon v$

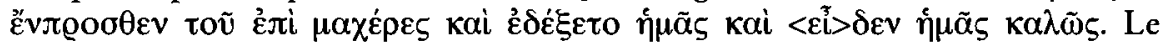
notariat de village lui-même pourrait devoir quelque chose à l'armée: c'est ce que suggère en tout cas le terme de librarius qu'on trouve à Beth Phouraia $(9,33)$ pour désigner un tabellion alors qu'à Marcopolis $(6,40)$ le notaire porte la dénomination civile de vouıкó $\varsigma^{38}$.

En somme, un peu comme le Hawrān récemment étudié par J. D. Grainger, un monde rural, peu urbanisé, sans structures d'autoadministration de droit public, encore moulé dans ses cantons préromains et porté à bout de bras par l'administration militaire romaine $e^{39}$. Cependant, vers la fin de la période couverte par le dossier, cette société entre dans le grand mouvement de la municipalisation. Et c'est du reste un des apports historiques majeurs du dossier qu'en si peu d'années, il nous fasse assister à la disparition du royaume d'Osrhoène, à la transformation de Hayklā-Karkāa de Sìdā „du roi Abgar" en Marcopolis et à la municipalisation d'Appadana .

Ce dernier événement a dû modifier les rapports entre la campagne et le cheflieu, entre les civils et l'administration militaire, à l'avantage de la ville et des civils. On mesure en partie d'après la pétition 3-4 l'incidence de cette mutation institutionnelle sur la vie de village. Ce texte a été soumis par un villageois de Beth Phouraia appelé Abidsautas fils d'Abidierdas qui se déclare, du nom de la nouvelle cité,

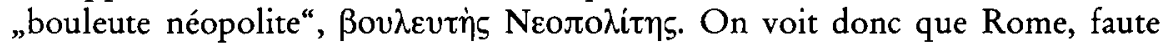
sans doute de notables suffisamment nombreux et fortunés à Appadana même, a dû remplir de campagnards du territorium l'album sénatorial de Néapolis, ce qui en dit long sur la faiblesse de la vie urbaine antérieure dans la région. Abidsautas a continué du reste à vivre à Beth Phouraia, comme on l'a remarqué pour des sénateurs de Bostra ${ }^{40}$. Néanmoins, il ne garde pas sa formule d'origo d'autrefois; il

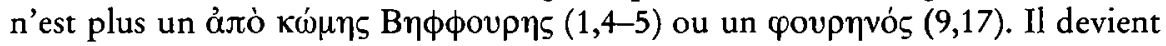
un „résident" de son ancien village, oikôv. Ainsi la société villageoise a-t-elle été

38 On a aussi librarius en Arabie d'après les P.Yadin. Nous écartons peut-être un peu rapidement une origine militaire dans notre édition sous presse des contrats de vente-achat (voir Naphtali Lewis, Two Greek Documents from Provincia Arabia, in: Illinois Classical Studies 3 [1978] 105).

39 Jobn J. Grainger, "Village Government" in Roman Syria and Arabia, in: Levant 27 (1995) 179-195, sp.192-193. Grainger ne parait pas avoir compris le rôle du centurion et autres officiers de police qu'il juge informel (p. 184).

40 Maurice Sartre, Bostra des origines à l'Islam (Paris 1985) 79. 
scindée en un groupe continuant à mener son genre de vie traditionnel, mais placé désormais sous la coupe des instances municipales civiles de Néapolis, et un groupe de notables promus, tournant le dos à leurs anciens convicani, ov $\gamma$ $\kappa \omega \mu \tilde{\eta} \tau \alpha^{41}$, ayant rompu le lien communautaire villageois et s'acquittant désormais de leurs munera et revêtant les charges municipales à la ville. Mais les ravages subis par la région en 256, à preuve le destin de Doura, ont été radicaux, si bien que la transformation sociale et institutionnelle qui s'amorçait a tourné court.

\section{La pétition de Bostra (P. Bostra 1; 29 mai 260)}

L'édition des deux papyrus de Bostra n'est pas encore prête, à cause des graves difficultés de lecture de P. Bostra 2 (contrat de mariage) ${ }^{42}$. Mais l'existence de ces document est déjà bien connue ${ }^{43}$, des copies en circulent et beaucoup de spécialistes sont impatients de pouvoir s'y référer dans leurs travaux. J'ai cru répondre en partie à leur attente en en publiant ici d'une manière très succincte le texte de la pétition P. Bostra $1^{44}$.

A part l'absence d'une formule de salutation (comme dans 5) cette pétition présente les caractères diplomatiques relevés dans les "libelles“ syriens et qui sont pour la plupart ceux des pétitions en général. La souscription latine indéchiffrée de l'officier de police évoque celle du centurion dans 5 . Toutefois, dans le papyrus de Bostra, la date a été apposée par le scribe grec et non pas par l'officier de police comme dans la pétition de l'Euphrate.

Une dame Aurelia Thopheisè ${ }^{45}$, du village d'Azzeira, relevant du district de l'Aianitide de la colonie auguste et métropole de Bostra ${ }^{46}$, se plaint à l'officier de police $\mathrm{du}$ ressort stationné à .ôba, d'un détournement d'argent et de documents ( $\delta \iota \kappa \alpha \iota \omega \mu \alpha \tau \alpha)$ par son neveu Sadeilos survenu pendant la maladie et l'agonie à Bostra de Kassianos son petit-fils. Thopheisè fait aussi état d'un deuxième grief. Dans

41 C'est le terme qu'on a en 1 et 4 , qu'on ne trouve du reste que dans le grec d'Orient et qui paraît impliquer quelque forme de solidarité communautaire.

42 Cotton, Cockle, Millar, Papyrology $223 \mathrm{n}^{\circ} 172$.

43 Voir Cotton, Cockle, Millar, Papyrology $223 \mathrm{n}^{\circ} 171$.

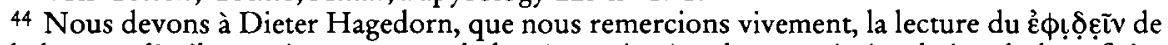
la 1.23 et d'utiles conjectures pour la $1.1(\sigma v \mu \mu \alpha \dot{\alpha} \chi(\omega)$ et la souscription latine du bénéficier (1. 32).

${ }^{45}$ Le $\varepsilon$ de ce nom est douteux, mais la ligature du signe en cause avec le $\iota$ est plus caractéristique du groupe $\varepsilon$ que de $\alpha$.

46 Pour la formule d'origo, avec mention de ressorts intermédiaires entre le village et la civitas, insolite en Égypte, comparer P.Yadin 16, 13-14. Pour le terme d'öpıov dans les formules

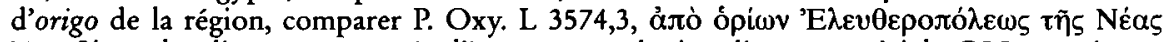
'Apaßíaร; plus directement, et régulièrement avec le singulier comme ici, les P.Ness. qui proviennent d'un territoire originellement „arabe $e^{\star}$. D'après des papyrus byzantins exhumés récemment à Petra, Bostra et Petra partageaient le même protocole colonial (dont le titre insolite d'Augoustokolonia). 
ces circonstances pénibles, un de ses bergers a détourné du cheptel. Elle demande à l'officier que les personnes incriminées soient déférées au centurion de police chargé, comme dans 5, de l'ev̉̃a $\xi \hat{i} \alpha$, dans l'attente d'une action en justice. La demande de l'intéressée a été acceptée comme en fait foi la souscription latine (indéchiffrée actuellement) et la pétition a été ensuite classée aux archives comme le montre l'endossement qui fait état du lieu, Azzeira avec peut-être la mention de l'agent de police impliqué, le beneficiarius.

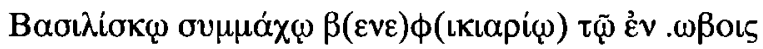

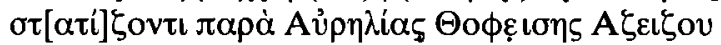

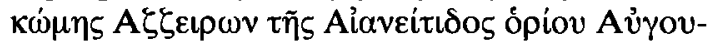

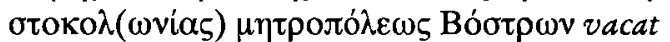

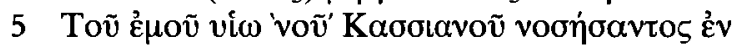

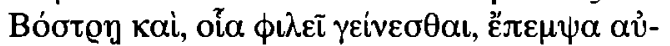

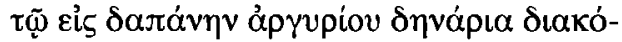

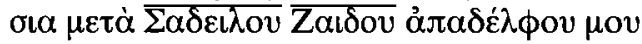

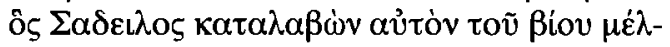

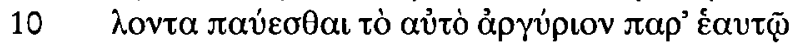

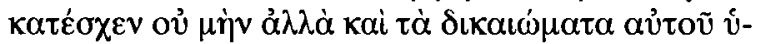

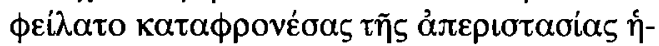

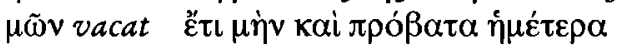

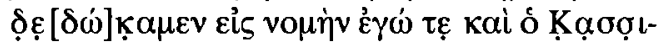

15 avò

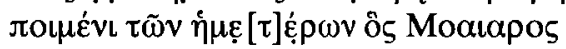

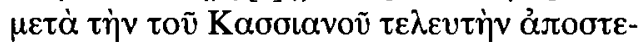


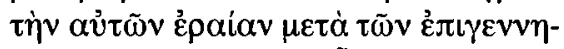

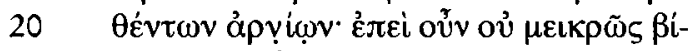

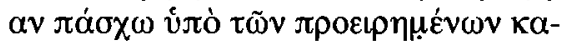

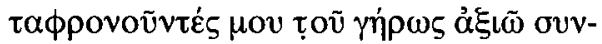

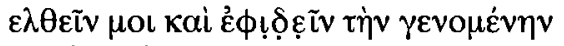

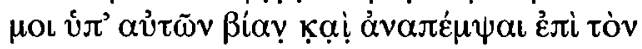

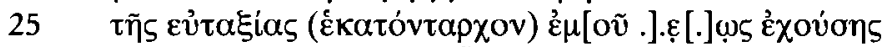

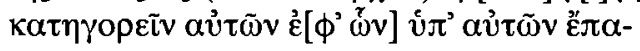
Өov

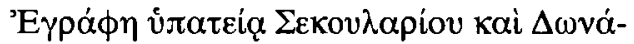

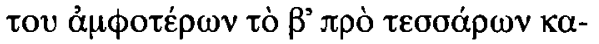

$30 \lambda \alpha v \delta(\tilde{\omega} v)^{\prime} \mathrm{I}$ Iovvi $\omega v$

vacat

fi ...ur

verso

$\mathrm{A} \zeta \zeta \varepsilon \iota p[\omega] \nu \ldots \beta$ 


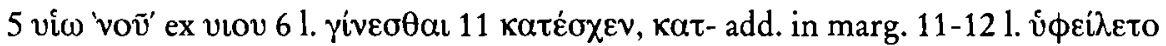

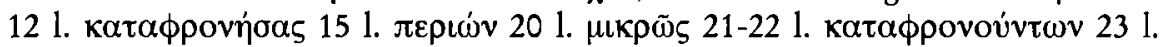

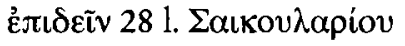

„A Basiliskos auxiliaire (?) 47 beneficiarius stationné à (.)oba, de la part d'Aurelia Topheisè, flle d'Azeizos, du village d'Azzeira de l'Aianitide des confins de Bostra, colonie auguste et métropole.

„Mon petit-fils Kassianos étant tombé malade à Bostra, comme c'est l'usage, je lui ai envoyé pour ses frais deux cents deniers d'argent par l'intermédiaire de Sadeilos fils de Zaeidos mon neveu (ou cousin). Lequel Sadeilos, tombant sur lui au moment où il allait cesser de vivre, garda cet argent par devers lui, et non content de cela, il s'empara de ses papiers, méprisant notre isolement. Bien plus, nous avions donné nos moutons à faire paître, moi ainsi que Kassianos vivant encore, à Moaiaros fils de Rabeibèlos, un de nos bergers. Lequel Moaiaros, après le décès de Kassianos, veut m'enlever ces miennes bêtes, leur laine avec le croît des agneaux. Attendu donc, que je n'endure pas une mince violence de la part des susdites personnes qui méprisent ma vieillesse, je te demande de venir à mon aide, de constater la violence commise par eux contre moi et de les déférer auprès du centurion chargé du maintien de l'ordre, car je suis (prête? $)^{48}$ à intenter une action contre eux pour ce que j'ai souffert par eux."

Ecrit sous le consulat de Saecularius et de Donatus, tous les deux consuls pour la deuxième fois, le quatre des calendes de juin"

[souscription latine]...

[au verso] ( $A$ ?) Azzeira, (de la part du?) beneficiarius (?)“.

47 Il se présente ici une sérieuse difficulté institutionnelle qui sera traitée dans l'édition.

48 On ne peut lire aisément ici un adverbe comme étoíl $\omega$ s vel sim. suggéré pourtant par 3-4 et 5 ou des pétitions égyptiennes du Bas-Empire. 
